\title{
Architectural Design Criteria for f-Block Metal Sequestering Agents
}

\author{
Pacific Northwest National Laboratory \\ June 1,1997 \\ Progress Report
}

Principal Investigator

Dr. B.P. Hay

(509) 372-6239 (Phone)

bp_hay@pnl.gov

Pacific Northwest National Laboratory

PO Box 999, MSIN K9-77

Richland, WA 99352
PNNL Contributors

Dr. M. Alnajjar

Dr. D.A. Dixon

Dr. G.J. Lumetta

Dr. L. Rao

Dr. B.M. Rapko

\section{External Collaborators}

Prof. R.T. Paine

(505) 277-1661 (Phone)

rtpaine@unm.edu

Department of Chemistry

University of New Mexico

Albuquerque, NM 87131

Prof. K.N. Raymond

(510) 642-7219 (Phone)

raymond@garnet.berkeley.edu

Department of Chemistry

University of Calfomia at Berkeley

Berkeley, CA 94720

Prof. D.M. Roundhill

(806) 742-3067 (Phone)

uldmr@ttacs.ttu.edu

Department of Chemistry

Texas Tech University

Lubbock, TX 79409- 1061
AWU Contributors

Dr. O. Clement

Dr. B.K. McNamara

Dr. G. Sandrone

Dr. P.L. Zanonato 


\section{Research Objective}

The objective of this project is to provide the means to optimize ligand architecture for f-block metal recognition. Our strategy builds on an innovative and successful molecular modeling approach in developing polyether ligand design criteria for the alkali and alkaline earth cations. The hypothesis underlying this proposal is that differences in metal ion binding with multidentate ligands bearing the same number and type of donor groups are primarily attributable to intramolecular steric factors. We propose quantifying these steric factors through the application of molecular mechanics models.

The proposed research involves close integration of theoretical and experimental chemistry. The experimental work entails synthesizing novel ligands and experimentally determining structures and binding constants for metal ion complexation by series of ligands in which architecture is systematically varied. The theoretical work entails using electronic structure calculations to parameterize a molecular mechanics force field for a range of metal ions and ligand types. The resulting molecular mechanics force field will be used to predict low-energy structures for unidentate, bidentate, and multidentate ligands and their metal complexes through conformational searches. Results will be analyzed to assess the relative importance of several steric factors including optimal M-L length, optimal geometry at the metal center, optimal geometry at the donor atoms (complementarity), and conformation prior to binding (preorganization). An accurate set of criteria for the design of ligand architecture will be obtained from these results. These criteria will enable researchers to target ligand structures for synthesis and thereby dramatically reduce the time and cost associated with metal-specific ligand development.

\section{Research Statement}

Critical tasks in the cleanup of U.S. Department of Energy (DOE) sites include processing radioactive wastes for disposal in long-term storage, remediation/restoration of environmental sites resulting from radioactive contamination, and decontamination/decommissioning of nuclear facilities. Because the radioactive components, most of which are metals, are typically present in very low concentrations, it is desirable to remove them from the bulk of the contaminated source (process waste stream, groundwater, soil) and concentrate them to minimize the volume of radioactive material destined for permanent subsurface disposal and thus minimize further waste management and disposal costs. One group of radionuclides, the actinides, which are pervasive throughout the DOE complex, is of special concern. In particular, thorium, uranium, neptunium, plutonium, americium, and curium with half-lives ranging from $10^{2}$ to $10^{6}$ years can all be found in various DOE wastes, contaminated soils and groundwaters, and contaminated facilities. Lanthanide elements are also of concern because they form an important group of fission

products that can persist in radioactive wastes for decades (e.g., ${ }^{152,154,155} \mathrm{Eu}$ ). 
Organic ligands with a high degree of metal ion specificity are essential components for developing separations processes for metal ions. Over the past 50 years, much research has focused on the discovery of selective ligands for $f$-block metal separations; both neutral and ionic ligands have been examined.

Despite past success in the discovery of ligands that exhibit some degree of specificity for the $f$-block metal ions, the ability to further control binding affinity and selectivity remains a significant challenge to the synthetic chemist. The approach for developing these ligands has involved lengthy and costly experimental programs of organic synthesis and testing, which, in the absence of reliable methods for screening compounds before synthesis, requires an extensive research effort. Criteria for accurately selecting target ligands would result in much more effective use of resources.

\section{Research Progress}

This project uses a combined theoretical and experimental approach to develop ligand design criteria for selective complexation off-block metal ions. These criteria will address how to best connect donor functionality to achieve metal ion recognition in multidentate ligands. For the $f$ block metal ions, donor groups of interest include amides, phosphine oxides, pyridine $\mathrm{N}$-oxides, and catechols. In FY97 our research has focused on amide ligands with $35 \%$ of our resources allocated for theory and $65 \%$ of our resources allocated for experiment. Ligands containing the amide functional group are currently used in DIAMEX (actinide separation process under testing for treatment of commercial reprocessing wastes) and TRUEX (actinide separation process under testing for use in tank waste cleanup at Idaho National Engineering and Environmental Laboratory (INEEL)).

Our approach will use molecular mechanics models to relate ligand structure to metal ion binding affinity. To provide a firm foundation for this study, an exhaustive review on the application of molecular mechanics to obtain structure-function relationships in coordination chemistry has been written and accepted for publication as a chapter in the Encyclopedia of Computation Chemistry. This review covers all molecular mechanics methods that have been successful in correlating ligand structure with reactivity toward metal ions.

The use of a molecular mechanics model requires the development of an extended force field to handle metal-amide complexes. This will be accomplished using both experimental data (crystal structures and vibrational spectra) and electronic structure calculations. Ab initio DFT and molecular orbital theory calculations will be used to examine the structure and bonding of the various metal ions to simple unidentate and bidentate ligands. Because the design of selectivity involves both an affinity for the target metal and a rejection of competing metal ions, the MM3 model needs to be applicable not only to $f$-block metals but to other metals. For this reason, our model is being designed to evaluate amide complexes with metal ions throughout the periodic table. 
Force field development for metal-amide complexes is well underway. An exhaustive survey of the Cambridge Structural Database has been conducted. A review article on structural aspects of metal-amide interactions has been written and will be submitted for publication. The database survey yielded 60 metal amide crystal structures that have been used in parameter development. A preliminary set of MM3 parameters have been developed and will be refined on completion of electronic structure calculations (vide infra). The structures used in this parameterization contained a variety of metal ions including lanthanides and actinides. Parameter correlations with metal size and charge are under development and will be used to extend the model to metals not covered by the existing experimental data. An abstract covering the results of this study has been submitted for presentation at the Fall National ACS Meeting.

Electronic structure calculations on metal-amide complexes are in progress. These calculations will yield ground state geometries and potential energy surfaces that will be used to validate and refine the MM3 parameters obtained through empirical fitting to crystal structure data described above. Because of their expense, the electronic structure calculations are focused on simple complexes of representative metal ions selected to cover a range of charge, size, and metal electronic configuration. Density functional theory has been used to calculate the structures of a variety of amides and metal ion complexes at the local and nonlocal levels with at least polarized valence double zeta basis sets. Frequencies have been calculated for all species to determine if they are minima. The calculations have been done with the computer codes NWChem and UniChem. This work has provided extensive testing of the new code NWChem being developed at the Environmental Molecular Sciences Laboratory (EMSL), Pacific Northwest National Laboratory (PNNL).

The first set of DFT calculations centered on optimizing the structure of the simple amides N,N-dimethylacetamide (DMA), malonamide, tetramethylmalonamide and its enol, and tetramethylsuccinamide. Calculations have also been done on the complex of DMA with the ions $\mathrm{Mg}^{2+}, \mathrm{Ca}^{2+}, \mathrm{Sr}^{2+}, \mathrm{Cu}^{2+}$ and $\mathrm{Zn}^{2+}$. The results of binding the naked ions showed that the $\mathrm{C}(\mathrm{O})--\mathrm{M}^{2+}$ distances were too short as compared to experiment and that there were problems with the $\mathrm{Zn}^{2+}$ basis set for this type of structure. Attention is now focused on $\left[\mathrm{M}(\mathrm{DMA})\left(\mathrm{OH}_{2}\right)_{5}\right]^{\mathrm{nt}}$ species. These complexes were generated from optimized structures of hexa-aquo complexes for the dications above as well as $\mathrm{Mn}^{2+}, \mathrm{Fe}^{3+}$ and $\mathrm{K}^{+}$. For $\mathrm{Li}^{+}$and $\mathrm{Na}^{+}$, also under examination, the number of $\mathrm{H}_{2} \mathrm{O}$ molecules in the aquo complex is 4 and 5, respectively. Comparison to experiment or the MP2 calculations of Feller show good agreement for the pure aquo clusters. The $\mathrm{C}(\mathrm{O})---\mathrm{M}^{2+}$ distances are in much better agreement with the values from the crystal structures. Geometries have been optimized for the penta-aquo dication metal complexes with DMA. Potential curves for the $\mathrm{C}(\mathrm{O})---\mathrm{M}$ stretch, the C-O-M bend, and the torsion about the C-O bond have been calculated for the $\mathrm{MgZ}^{2+}$ derivative and are in progress for the other four dications. When these calculations are complete, we will do the same for the three alkali ions noted above, the trications $\mathrm{Fe}^{3+}, \mathrm{Sc}_{+}^{3}, \mathrm{La}^{3+}$, and $\mathrm{Lu}^{3+}$, and the tetracations $\mathrm{Ti}^{4+}, \mathrm{Zr}^{4+}$, and $\mathrm{Th}^{4+}$. The DFT calculations on the $f$-block metal ion species will be done with effective core potentials, calculations that can only be done with our new program, NWChem.

Experimental structure-stability data is needed to validate predictions from theoretical models. Such sets of data would satisfy the following criteria: 1) the ligand series should differ 
only in the placement of the donor atoms, 2) the coordination environment of the metal-ligand complexes must be known, and 3) relative binding affinities should be determined under identical experimental conditions of solvent, counter ion, and temperature. A literature search was performed to locate any information regarding single-phase stability constants for such amide complexes of lanthanides or actinides and solution structural information on such complexes. This search revealed that not only are there no existing structure-stability data sets, but that there are no stability-constant data for $f$-block metal ions with pure amide ligands. Given that amide group is neutral over a wide $\mathrm{pH}$ range, this situation reflects the general difficulty of determining stability constants for non-ionizable ligands. The goal of this year's experimental effort is to generate structure-stability data sets for series of ligands bearing only the amide functionality with selected $f$-block metal ions. To achieve this goal, experimental effort has focused on (1) amide ligand synthesis and (2) evaluation of known methods for the determination of metal-amide binding constants.

A series of twelve diamides based on derivatives of malonamide and succinamide has been targeted for synthesis at PNNL. These ligand structures have been chosen to examine the influence of the spacing between the two amide groups, alkyl substitution on the methylene or ethylene spacer, and N-alkyl substitution. Five of the twelve diamides have been prepared in tens of gram quantities in $99+\%$ purity. Purity and composition have been determined by proton and carbon NMR, GC-MS, and FTIR. The remaining seven diamides are scheduled to be completed by the end of FY97. In addition, a subcontract has been placed with Professor D. Max Roundhill at Texas Tech University to prepare series of calix[4]- and calix[6]-arene amides. Synthetic routes to four compounds have been demonstrated and scale-up to multi-gram quantities is in progress. Synthetic routes to other compounds in these series are under development.

A variety of methods for the determination of metal-amide binding constants are under investigation. These methods include electronic spectroscopy, IR spectroscopy, use of ion selective electrodes, calorimetry, and solvent extraction. Of these methods, only solvent extraction remains to be tried.

Electronic spectroscopy has been used to monitor the complexation of DEHBA (N,N-di-2ethylhexyl)butanamide) and the DIAMEX ligand (N,N'-dimethyl-N-N'dibutyl-2-tetradecylpropane-1,3-diamide) with $\mathrm{Eu}(\mathrm{III})$ and $\mathrm{Nd}$ (III) in acetonitrile. This method is not applicable for these metal ions because there is very little shift in absorption bands on amide complexation. Another electronic spectroscopy approach is to monitor competition of the amide ligand with a ligand that absorbs strongly in the visible region, e.g., murexide. However, this approach failed in the case of TMM (N,N,N'N'-tetramethylmalonamide) with Eu(III). Although neither approach appears suitable for lanthanides, the possibility of using electronic spectroscopy methods for actinides remains to be investigated. The amide ligands are known to extract the tetravalent and hexavalent actinides better than the trivalent lanthanides, which suggests stronger binding of the amides to the former metal ions. Thus, it is expected that amide binding the tetravalent and hexavalent actinides will result in significant shifts in the electronic spectra of these ions. 
The frequency of the intense carbony1 stretch is known to be sensitive to metal ion complexation. Therefore, FTIR spectroscopy was used to examine the complexation of $\mathrm{Eu}(\mathrm{III})$ with DEHBA and the DIAMEX ligand. Although shifts in the carbonyl frequency of $40-50 \mathrm{~cm}^{-1}$ were observed in the presence of $\mathrm{Eu}(\mathrm{III})$, the spectra are complex. The FTIR spectra indicate the formation of two or more different Eu/amide complexes depending on the Eu/amide ratio. The presence of multiple species vitiates the determination of binding constants by the continuous variation method. It remains to be seen whether the calixarene/amide systems will also form multiple species when reacted with lanthanide and actinide ions.

Because ion-selective electrodes specific for $f$-elements are not available, the measurement of metal-amide binding constants by potentiometry requires the use of a competitor, i.e., another metal ion in solution that competes with the f-block metal ion and whose concentration can be determined by a reversible electrode. Such electrodes are available for $\mathrm{Ag}(\mathrm{I}), \mathrm{Cd}(\mathrm{II})$, and $\mathrm{Pb}(\mathrm{II})$. Potentiometric measurements of $\mathrm{Ag}(\mathrm{I})$ in absence and presence of the DIAMEX ligand indicate that the interaction between this metal ion and the ligand are too weak to be observed in acetonitrile solvent. The possibility of using $\mathrm{Cd}(\mathrm{II})$ or $\mathrm{Pb}(\mathrm{II})$ as a competitor remains to be investigated.

To date the most promising approach has been calorimetry. The complexation of Eu(III) by both the TMM and the DIAMEX ligands have been investigated in a number of pure and mixed solvents. In a solvent that coordinates the metal ion strongly (e.g., DMSO), no complexation is detected. In a solvent that coordinates the metal ion weakly (e.g., acetonitrile or propylene carbonate), complexation is too strong ( $\log \mathrm{K}$ greater than 5$)$ to be measured by this method. We have demonstrated that mixtures of weak and strong solvents allow binding constants to be measured by this method. For example, we have been able to determine a 1:1 binding constant $(\log \mathrm{K}=1.2)$ and a $1: 2$ binding constant $(\log \mathrm{K}=2.3$ ) for complexation of Eu(III) with TMM in 90\% acetonitrile: 10\% DMSO. To our knowledge this is the first determination of a lanthanide diamide binding constant. The data demonstrate that the solvent can be adjusted to bring binding constants within the range that can be measured by this method. Alternative solvent systems, with metal coordinating properties intermediate to the extremes investigated to date, are under investigation. We are also exploring the possibility of measuring the heats of formation for the metal/amide complexes directly by using thermochemical methods such as thermogravimetric analysis, differential scanning calorimetry, and bomb calorimetry. This latter approach has the advantage that solvent effects are eliminated.

Although our major emphasis has been on amides, research also has begun on other classes of ligand within the scope of this project. These include mixed amide-phosphine oxide, mixed phosphine oxide-pyridine $\mathrm{N}$-oxide, and catechol ligands.

A solvent extraction method has been used to determine single-phase binding constants of the TRUEX ligand ((octyl)(methyl)-N,N-diisobutyl-carbamoylmethylphosphine oxide) and NOPOPO ((2,6-bis(diphenylphosphino)methylpyridine N,P,P trioxide) with Am(III) and Eu(III). This is the first measurement of a binding constant for the TRUEX ligand. The results of this study show NOPOPO is a much more potent actinide extractant than the TRUEX ligand and demonstrate that the degree of actinide extraction correlates with single-phase binding constants. 
A manuscript on the binding constant measurements has been written and will be submitted for publication. A second manuscript on the distribution of $\mathrm{Am}(\mathrm{III}), \mathrm{Eu}(\mathrm{III})$, and $\mathrm{UO}_{2}$ (II) with NOPOPO is in preparation.

We have recently received a set of structure-stability data for $\mathrm{Fe}(\mathrm{III})$ complexation with a series of tris-catecholate ligands compiled by Prof. Kenneth N. Raymond at the University of California - Berkeley. This data will be useful in the parameterization and validation of MM3 force field parameters for metal-catechol complexes.

\section{Presentations}

Rapko, B.M. "Extraction off-Elements by Phosphine Oxide/Pyridine N-Oxide Ligands." 213th ACS National Meeting, San Francisco, CA, April 13 - 17th, 1997.

Lumetta, G.J., B.K. McNamara, and I.E. Burgeson. "Amide Complexes off-Block Elements." Abstract submitted for the 21st Annual Actinide Separations Conference, Charleston, SC, June 23-26, 1997.

Rao, L., Y. Xia, B.M. Rapko, and P.L. Martin. "Synergistic Extraction of Eu(III) and Am(III) by TTA and the Neutral Donor Extractants CMPO and NOPOPO." Abstract submitted for the 21st Annual Actinide Separations Conference, Charleston, SC, June 23-26, 1997.

Zanonato, P.L., and L. Rao. "Complexation of Eu(III) by N,N,N',N'-tetraalkyldiamides." Abstract submitted for the 214th ACS National Meeting, Las Vegas, NV, September 7-11, 1997.

Clement, O., G. Sandrone, D.A. Dixon, and B.P. Hay. "A MM3(96) Force Field for MetalAmide Complexes." Abstract submitted for the 214th ACS National Meeting, Las Vegas, NV, September 7-11, 1997.

Rapko, B.M., G. J. Lumetta, B.K. McNamara, L. Rae, and P.L. Zanonato. "Determination of Actinide and Lanthanide Binding Constants with Amides and Diamides." Abstract submitted for the Tenth Symposium on Separation Science and Technology for Energy Applications, October 20-24, Gatlinburg, TN. 


\section{Publications}

Hay, B.P., and O. Clement. "Metal Complexes" in Encyclopedia of Computational Chemistry, P. von Rague Schleyer, Editor-in-Chief, John Wiley and Sons, London, 1997, in press.

Clement, O., and B.P. Hay. "Structural Aspects of Metal-Amide Complexes." Manuscript in preparation for Coordination Chemistry Reviews.

Dixon, D.A., and G. Sandrone. "The Structures and Energetics of Aquo-Metal Dication Complexes from Density Functional Theory." Manuscript in preparation for publication in Journal of Physical Chemistry.

Sandrone, G., O. Clement, B.P. Hay, and D.A. Dixon. "The Structures and Energetics of Metal Dication-Amide Complexes from Density Functional Theory." Manuscript in preparation for publication in Journal of Physical Chemistry.

Rao, L., Y. Xia, B.M. Rapko, and P.F. Martin. "Synergistic Extraction of Eu(III) and Am(III) by Thenoyltrifluoroacetone and Neutral Donor Extractants: Octyl(Phenyl)- N,NDiisobutylcarbonyl-methylphosphine Oxide and 2,6-Bis(Diphenylphosphino)methyl Pyridine N,P,P Trioxide." Manuscript in preparation for publication in Solvent Extraction and Ion Exchange.

Rapko, B.M., G.J. Lumetta, B.K. McNamara, L. Rao, and P.L. Zanonato. "Determination of Actinide and Lanthanide Binding Constants with Amides and Diamides." Manuscript in preparation for publication in Separation Science and Technology. 